\title{
Multi-Mode Coupling Wave Theory for Helically Corrugated Waveguide
}

\author{
Liang Zhang, Wenlong He, Kevin Ronald, Alan D. R. Phelps, Colin G. Whyte, Craig W. Robertson, \\ Alan R. Young, Craig R. Donaldson, and Adrian W. Cross
}

\begin{abstract}
Helically corrugated waveguide has been used in various applications such as gyro-backward wave oscillators, gyro-traveling wave amplifier and microwave pulse compressor. A fast prediction of the dispersion characteristic of the operating eigenwave is very important when designing a helically corrugated waveguide. In this paper, multi-mode coupling wave equations were developed based on the perturbation method. This method was then used to analyze a five-fold helically corrugated waveguide used for X-band microwave compression. The calculated result from this analysis was found to be in excellent agreement with the results from numerical simulation using CST Microwave Studio and vector network analyzer measurements.
\end{abstract}

Index Terms-Coupling coefficients, coupling wave theory (CWT), dispersion curve, helically corrugated waveguide.

\section{INTRODUCTION}

$\mathbf{S}$ TRAIGHT hollow metal waveguides with uniform cross section can only support modes whose phase velocity is larger than the speed of light, while waveguides with periodic corrugations can propagate modes with phase velocity less than the speed of light and are suitable for particle beam-wave interactions. Periodically corrugated waveguides have been used in many applications, including slow-wave structures with axial periodicity for conventional backward wave oscillators (BWOs) and traveling wave tubes (TWTs)[1], and magnetrons [2] with azimuthal periodicity. Corrugated waveguide structures have also been used as Bragg reflectors [3], [4]. The helically corrugated waveguide, which contains both axial and azimuthal periodicity, has attracted significant interest in the last 10 years and has successfully found applications in the gyrotron traveling wave amplifier (gyro-TWA) [5], [6], as a dispersive medium for frequency-swept microwave pulse compression[7], [8] and in the gyrotron backward wave oscillator (gyro-BWO)[9], [10]. The electrodynamic properties of the helically corrugated waveguide are important, as the dispersion characteristic of the operating eigenwave can be changed by

Manuscript received March 28, 2011; revised September 13, 2011; accepted September 20, 2011. Date of publication November 02, 2011; date of current version December 30, 2011. This work was supported by the U.K. EPSRC Research Grant EP/E058868/1. The work of L. Zhang was supported by SUPA and SORSAS under the award of a SUPA prize research studentship.

The authors are with SUPA, Department of Physics, University of Strathclyde, Glasgow, G4 0NG, Scotland, U.K. (e-mail: liang.zhang@strath.ac.uk; w.he@strath.ac.uk; k.ronald@strath.ac.uk; a.d.r.phelps@strath.ac.uk; colin. whyte@strath.ac.uk; craig.robertson@strath.ac.uk; a.r.young@strath.ac.uk; craig.donaldson@strath.ac.uk; a.w.cross@strath.ac.uk).

Color versions of one or more of the figures in this paper are available online at http://ieeexplore.ieee.org.

Digital Object Identifier 10.1109/TMTT.2011.2170848 choosing different corrugation depths and corrugation periods to meet the needs of different applications. In a gyro-TWA or gyro-BWO, a constant group velocity in the operating frequency range is desired, while in a microwave compressor, a linearly varying group velocity in the frequency band is preferred.

Several methods have been developed to investigate the dispersion characteristics of the helically corrugated waveguide by analytical and numerical techniques. The simplest and fastest method to calculate the dispersion curves is the coupled wave theory based on the method of perturbation [11]-[13]. It assumes that the change in the cross section is small and can be treated as a first order derivative of a regular cross section. Reference [12] gives the dispersion relation between two TE modes and [11] gives the results of the coupling coefficients between two TE modes, two TM modes, and one TE mode with one TM mode but no derivations were presented. It is found that when the corrugation depth is less than $15 \%$ of the mean radius of the waveguide, the coupled wave theory gives reasonably accurate results. The coupled wave theory played an important role in the preliminary design of the three-fold helical waveguide in which the dispersion of the operating eigenwave is simply a result from the coupling between two TE modes in the operating frequency range, i.e., the spatial harmonic $\mathrm{TE}_{11}$ mode and $\mathrm{TE}_{21}$ mode. However, the three-fold helical waveguide has limited power capability. A helical waveguide working with a higher eigenwave mode can achieve higher power capability as a larger radius can be used. However operating with higher mode means more modes would take part in the coupling and therefore the resultant dispersion curve would be more complicated to calculate. For example, in a five-fold helical waveguide the spatial harmonic of the $\mathrm{TE}_{31}$ mode would couple with the $\mathrm{TE}_{21}, \mathrm{TE}_{22}$, $\mathrm{TM}_{21}$ and $\mathrm{TM}_{22}$ modes. The coupled mode equation, which only considers two TE modes, would therefore not be accurate in obtaining the dispersion characteristics of the five-fold helical waveguide. Necessary modifications to include the coupling between TE and TM modes are required and will be presented in this paper.

The dispersion curve of the helically corrugated waveguide can also be numerically simulated by using some computer codes, such as the finite-element method (FEM), the eigensolver in CST Microwave Studio, the electromagnetic field solver in the particle-in-cell (PIC) code MAGIC and the transient solver in CST Microwave Studio, which use a finite-difference time-domain method (FDTD) [14]. By using these codes, accurate results can be obtained if the mesh grid is dense enough. However, these 3-D simulations have the disadvantage of requiring long computational times to complete the simulations, 
for example, two or three days on a powerful desktop PC using a reasonably dense mesh grid and they are therefore not suitable for optimizing the dimensions of the helical waveguide for a particular application.

It should be noticed that the helically corrugated waveguide can also be regarded as a type of twisted waveguide. A helicoidal coordinate transform can be employed to convert the twisted waveguide into a straight uniform waveguide, thus downgrading the 3-D problem into a 2-D one [15]. The price of using this technique is the uniform material properties in the twisted waveguide will become non-uniform and position dependent. This transformation method can greatly reduce the computing time in resolving the eigenwave dispersion when applying the FEM and FDTD methods in the 2-D geometries [16], [17]. It should be noted that the dispersions of the coupled waves and those initial partial waves are all existing and calculated at the same time when this method is used. The dispersion curves of these modes would be interweaved and very close to each other, especially when a higher modes are considered. Therefore the dispersion curves of the operating eigenwaves of the helically corrugated waveguide would be difficult to identify. The field patterns of the eigenmodes can be helpful when identifying the operating dispersion curve, however, it is very difficult to recognize by computer automatically, thus it is not suitable for the optimization routing. On the other hand, this operating eigenwave from the transformation method can be easily identified by using the calculated result from the coupled wave theory as a reference.

It is evident that the analytical calculation using the coupled wave theory is very useful in the preliminary design of the helically corrugated waveguide. It is also useful to accurately and rapidly predict the dispersion of the eigenwave when used in combination with the transformation method. In this paper, a detailed description of the coupling coefficient between TE modes and TM modes and the extended eigenwave equations caused by multi-mode coupling are discussed in Section II. Dispersion calculation of a five-fold helically corrugated waveguide is described in Section III. The comparison between the theoretical calculation and the experiment is presented in Section IV.

\section{PRINCIPLE OF The Coupled WaVE TheORY}

The equation of the helical profile of the inner surface in a cylindrical coordinate system $(r, \theta, z)$ can be written as

$$
r(\theta, z)=R_{0}+R_{1} \cos \left(m_{B} \theta+\frac{2 \pi z}{d}\right)
$$

where $R_{0}$ is the mean radius of the circular waveguide, $R_{1}$ is the corrugation depth, $m_{B}$ is the fold number, and $d$ is the axial period of the corrugation. When the corrugation depth is not zero, two modes will couple when their axial and azimuthal wave numbers satisfy the synchronism conditions

$$
h_{1}-h_{2}=\frac{2 \pi}{d}, m_{1}-m_{2}=m_{B}
$$

where $h_{1}$ and $h_{2}$ are the axial wavenumbers of modes 1 and 2, and $m_{1}$ and $m_{2}$ are the azimuthal indices of modes 1 and 2 , respectively.

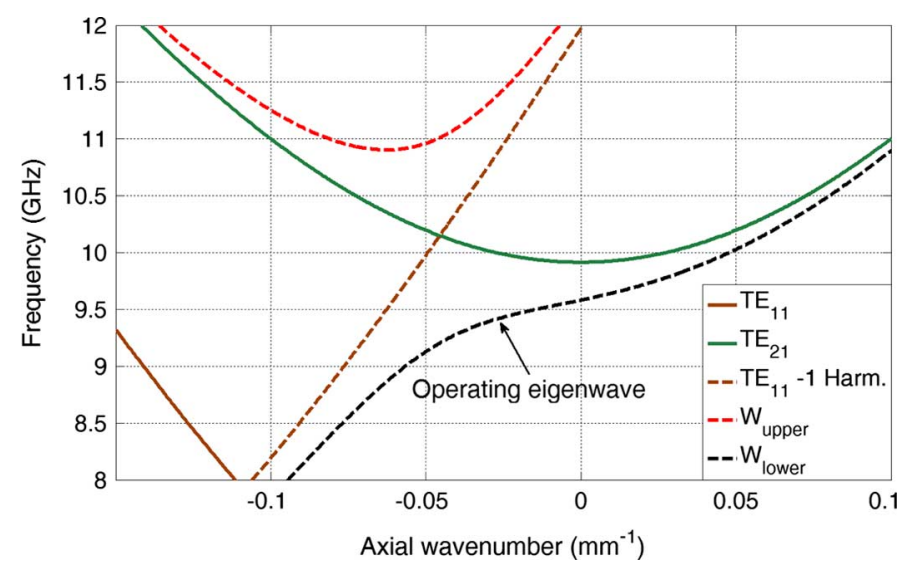

Fig. 1. Coupling between the $\mathrm{TE}_{21}$ mode and the spatial harmonic $\mathrm{TE}_{11}$ mode in a three-fold helical waveguide.

In three-fold helically corrugated waveguide, the $\mathrm{TE}_{21}$ mode would couple with the $\mathrm{TE}_{11}$ circularly polarized mode of opposite rotation and generate an operating eigenwave, as shown in Fig. 1. From the synchronism condition, coupling between higher modes requires larger fold number $m_{B}$. However, with larger $m_{B}$ more modes would satisfy the synchronism condition and they would couple with each other and hence contribute to the dispersion characteristic.

\section{A. Coupling Coefficients}

The coupling coefficient is the basis of the coupled wave theory as it indicates how strong the coupling between two modes is. In this paper, we start from the general equations of the coupling coefficients between two TE modes, two TM modes and one TE (mode $m$ ) to one TM (mode $j$ ) modes as shown in (3). The derivation of these equations can be found in [18], which contains a detailed introduction to the coupled mode theory

$$
\begin{aligned}
S_{j m}= & \frac{1}{2 h_{j}\left(h_{j}-h_{m}\right)} \oint v(s) \\
& \times\left[\alpha_{j}^{2} \alpha_{m}^{2} \Psi_{j} \Psi_{m}+\left(h_{j} h_{m}-k^{2}\right) \frac{\partial \Psi_{j}}{\partial s} \frac{\partial \Psi_{m}}{\partial s}\right] d s \\
S_{j m}= & \frac{k^{2}-h_{j} h_{m}}{2 h_{j}\left(h_{j}-h_{m}\right)} \oint v(s) \frac{\partial \phi_{j}}{\partial u} \frac{\partial \phi_{m}}{\partial u} d s \\
S_{j m}= & -\frac{k}{2 h_{j}} \oint v(s) \frac{\partial \phi_{j}}{\partial u} \frac{\partial \Psi_{m}}{\partial s} d s
\end{aligned}
$$

where $v(s)$ is the function to describe the difference between the perturbed non-uniform waveguide and the uniform waveguide. When $v(s)=0$, there is no coupling between the two modes. $h_{j}, h_{m}$ are the axial wave numbers of the coupled modes and $k$ is the free-space wave number. $\alpha_{j}, \alpha_{m}$ are the transverse wave numbers of modes $j$ and $m . \Psi, \varphi$ are the transverse eigenfunctions of the TE and TM modes of the unperturbed regular waveguide. $u$ is the normal vector directed towards the waveguide wall. $s$ is chosen so that axes $u, s, z$ to form a right-handed coordinate system. 
In circular waveguide, the transverse wave number and eigenfunction in a cylindrical coordinate system are in the form of

$$
\begin{aligned}
\alpha_{n q}= & \begin{cases}\frac{\xi_{n q}}{R_{0}}, & \text { (for TE mode) } \\
\frac{\varsigma_{n q}}{R_{0}}, & \text { (for TM mode) }\end{cases} \\
\Psi_{n q}= & \sqrt{\frac{2}{\pi \varepsilon_{n}}} \frac{1}{\sqrt{\xi_{n q}^{2}-n^{2}}\left|J_{n}\left(\xi_{n q}\right)\right|} \\
& \times J_{n}\left(\alpha_{n q} r\right)\left\{\begin{array}{l}
\cos (n \theta) \\
\sin (n \theta)
\end{array}\right\} \\
\varphi_{n q}= & \sqrt{\frac{2}{\pi \varepsilon_{n}}} \frac{1}{\zeta_{n q} \mid J_{n}^{\prime}\left(\zeta_{n q}\right)} J_{n}\left(\alpha_{n q} r\right)\left\{\begin{array}{l}
\cos (n \theta) \\
\sin (n \theta)
\end{array}\right\} \\
\varepsilon_{n}= & \left\{\begin{array}{l}
2,(n=1) \\
1,(n \neq 1)
\end{array}\right.
\end{aligned}
$$

where $\xi_{n q}$ is the $q^{t h}$ zero of the derivation of $J_{n}$ and $J$ is the first kind of Bessel function. $\zeta_{n q}$ is the $q^{\text {th }}$ root of $J_{n}$. In practical applications, the operating mode in the helically corrugated waveguide always satisfies $n \neq 0$. As the radial number $q$ in the modes does not contribute to the following derivation, symbols $j$ and $m$ are used to denote the azimuthal number of the two coupling modes for simplicity.

The difference between the helically corrugated cross section and the circular waveguide at position $z=0$ can be written as $v(s)=m_{B} R_{1} \sin \left(m_{B} \theta\right)$. For a small corrugation, we have $v(s) \ll R_{0}$. As $v(s)$ is in terms of the first-order derivation of the mean radius, if only the first-order approximation of the coupling coefficient is taken into account then only the zero-order approximation of the other terms in (3) needs to be considered. In polar coordinates, we can simply make $d s=r d \theta$ and $d u=d r$. Substituting these into (3), we can get a simpler form of the coupling coefficients, as

$$
\begin{aligned}
S_{j m}= & \frac{R_{1}\left[\xi_{m}^{2} \xi_{j}^{2}+\left(h_{j} h_{m}+k^{2}\right) j m R_{0}^{2}\right]}{h_{j}\left(h_{j}-h_{m}\right) R_{0}^{3} \sqrt{\xi_{m}^{2}-m^{2}} \sqrt{\xi_{j}^{2}-j^{2}}} \\
& \times \oint \frac{m_{B}}{\pi} \sin \left(m_{B} \theta\right)\left\{\begin{array}{l}
\cos (m \theta) \\
\sin (m \theta)
\end{array}\right\}\left\{\begin{array}{l}
\cos (j \theta) \\
\sin (j \theta)
\end{array}\right\} d \theta \\
S_{j m}= & \frac{R_{1}\left(k^{2}-h_{j} h_{m}\right)}{R_{0} h_{j}\left(h_{j}-h_{m}\right)} \\
& \times \oint \frac{m_{B}}{\pi} \sin \left(m_{B} \theta\right)\left\{\begin{array}{l}
\cos (m \theta) \\
\sin (m \theta)
\end{array}\right\}\left\{\begin{array}{l}
\cos (j \theta) \\
\sin (j \theta)
\end{array}\right\} d \theta \\
S_{j m}= & -\frac{R_{1} k m}{R_{0} h_{j} \sqrt{\xi_{m}^{2}-m^{2}}} \\
& \times \oint \frac{m_{B}}{\pi} \sin \left(m_{B} \theta\right)\left\{\begin{array}{l}
\cos (m \theta) \\
\sin (m \theta)
\end{array}\right\}\left\{\begin{array}{l}
\cos (j \theta) \\
\sin (j \theta)
\end{array}\right\} d \theta .
\end{aligned}
$$

In the helically corrugated waveguide, only the modes that satisfy the synchronism conditions can couple with each other. Thus we have $j-m=m_{B}$. It should be noted that, in the prac- tical application, $m$ is always chosen as a negative value indicating an opposite rotating mode and hence $h_{m}$ also is a negative value. The integral in this equation will become unity if we apply the synchronism condition. Then the final coupling coefficients for the helically corrugated waveguide become

$$
\begin{aligned}
S_{j m} & =\frac{R_{1}\left[\xi_{m}^{2} \xi_{j}^{2}+\left(h_{j} h_{m}+k^{2}\right) j m R_{0}^{2}\right]}{h_{j}\left(h_{j}-h_{m}\right) R_{0}^{3} \sqrt{\xi_{m}^{2}-m^{2}} \sqrt{\xi_{j}^{2}-j^{2}}} \\
S_{j m} & =\frac{R_{1}\left(k^{2}-h_{j} h_{m}\right)}{R_{0} h_{j}\left(h_{j}-h_{m}\right)} \\
S_{j m} & =-\frac{R_{1} k m}{R_{0} h_{j} \sqrt{\xi_{m}^{2}-m^{2}}} .
\end{aligned}
$$

Equation (7) has the same form as the ones in [11], while the coupling coefficient in [11] has been normalized to $h_{j} h_{m}$.

\section{B. Coupled Mode Equations}

Although the coupling coefficient indicates the strength of the coupling between two modes, it is useful to know the strength of the coupling modes in the resultant eigenwave. They can be solved from the coupled mode equations [18] and the coupling coefficient, as

$$
\left\{\begin{array}{l}
\frac{d V_{m}}{d z}=\frac{-h_{j}}{h_{m}} S_{j m} \exp \left(-i\left(\sigma_{j}-\sigma_{m}\right)\right) V_{j} \\
\frac{d V_{j}}{d z}=S_{j m} \exp \left(i\left(\sigma_{j}-\sigma_{m}\right)\right) V_{m}
\end{array}\right.
$$

where $V_{m}, V_{j}$ are the normalized voltages of mode $m$ and $j$ in the helically corrugated waveguide, respectively, and $\sigma_{j / m}=$ $\int_{0}^{z} h_{j / m} d z$ is the phase of mode $j$ or $m$ in the waveguide, and $\sigma=\sigma_{j}-\sigma_{m}=\left(h_{j}-h_{m}\right) d$ is the phase difference between the two coupled modes in the waveguide with the length of one period $d$. $\sigma$ becomes $2 \pi$ if the synchronism conditions are applied.

The normalized voltages of mode $m$ and $j$ in the helically corrugated waveguide can be solved as

$$
\left\{\begin{array}{l}
V_{m}=-\left(\cos \kappa+\frac{i \pi}{\kappa} \sin \kappa\right) \\
V_{j}=\frac{\sqrt{\kappa^{2}-\pi^{2}}}{\kappa} \sin \kappa
\end{array}\right.
$$

where

$$
\kappa=2 \pi \sqrt{\left|h_{j} / h_{m}\right| S_{j m}^{2}+1 / 4} .
$$

They are completely determined by $\kappa$ which is dependent on the axial wavenumbers of the coupling modes and the coupling coefficient.

\section{Dispersion Curve Function}

The new dispersion curve caused by two-mode coupling will satisfy the following equation:

$$
f_{j}^{2}(h) \cdot f_{m}^{2}(h)=h_{j}^{2} h_{m}^{2} S_{j m}^{2}
$$

where $f_{j}, f_{m}$ are the dispersion relations of the coupling modes which are functions of the axial wavenumber $h$. For a TE/TM 


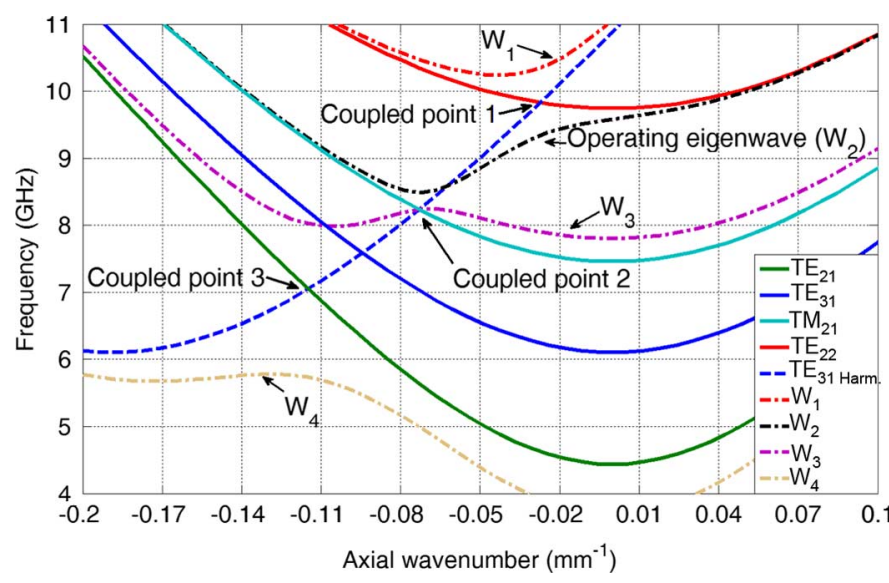

Fig. 2. Coupling modes in a five-fold helical waveguide.

mode in the circular waveguide, the dispersion relation can be written as

$$
f_{j}^{2}(h)=k^{2}-\alpha_{j}^{2}-h^{2} .
$$

Because, as noted in Section II-A, mode $m$ is the negative spatial harmonic wave, the dispersion curve is shifted to the left by $2 \pi / d$ to produce the following dispersion relation:

$$
f_{m}^{2}(h)=k^{2}-\alpha_{m}^{2}-\left(h-\frac{2 \pi}{d}\right)^{2} .
$$

Equation (10) is a fourth-order linear equation. Given a wave number $k$, we can get four eigenvalues by solving the equation. However, only two of them have practical physical meaning, and they are named as upper coupled mode $W_{\text {upper }}$ and lower coupled mode $W_{\text {lower }}$. If there is no coupling between the two modes, that is $S_{j m}=0$, the eigenfunction will degenerate into two separate dispersion curve functions, i.e., $f_{j}^{2}(h)=0$ and $f_{m}^{2}(h)=0$. The $W_{\text {upper }}$ and $W_{\text {lower }}$ will degenerate into the previous uncoupled partial eigenwaves.

There is no straightforward dispersion curve equation available that can take into account all of the considered modes for a helically corrugated waveguide operating in a higher mode. However, from the dispersion diagram, it is clear that the coupling frequencies between any two modes, as shown in Fig. 2 which shows the five-fold helical waveguide case, are different. Thus an alternative method is to calculate the coupled eigenwaves in sequence by using the two-mode coupling equation, either from the lower frequency to the higher frequency, or vice versa. Here the sequence from higher to lower frequencies is used. First, two eigenwaves $W_{\text {upper1 }}$ and $W_{\text {lower1 }}$ can be resolved from (10)-(12). Then eigenwave $W_{\text {upper1 }}$ does not have an intersection with the next mode, thus it is saved as an eigenwave ( $W_{1}$ in Fig. 2). Eigenwave $W_{\text {lower1 }}$ couples with the next mode to generate $W_{\text {upper2 }}$ ( $W_{2}$ in Fig. 2 ) and $W_{\text {lower2 }}$ by using (10), while here $f_{m}(h)$ refers to the eigencurve $W_{\text {lower } 1}$ and $h_{j}, h_{m}$ are the axial wavenumbers in the intersection of mode $W_{\text {lower1 }}$ and the next coupled mode. By repeating this process, all the eigenwaves caused by the mode coupling in the helically corrugated waveguide can be obtained.

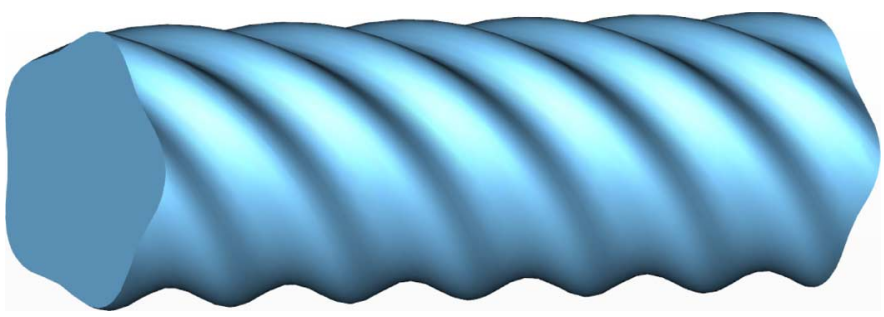

Fig. 3. Schematic view of the five-fold helical waveguide.

\section{Dispersion Calculation of a Five-Fold Helically CORRUGATED WAVEGUIDE}

One of the applications of the helically corrugated waveguide is as a dispersive medium for pulse compression. A three-fold corrugated waveguide operating in X-band was previously designed and was used in an experiment that achieved a compression factor of 25 [19]. The maximum power capability of the waveguide is about $1 \mathrm{MW}$. To pursue higher power capability [20], a five-fold helically corrugated waveguide with larger radius operating in the same frequency range has been investigated. A general schematic view of a five-fold helical waveguide is shown in Fig. 3. The desired coupled modes are the spatial harmonic $\mathrm{TE}_{31}$ mode and the $\mathrm{TE}_{22}$ mode. As known from the synchronism condition, besides the $\mathrm{TE}_{22}$ mode, the possible modes that can couple with the $\mathrm{TE}_{31}$ are the $\mathrm{TE}_{21}$ and $\mathrm{TM}_{21}$ modes in the lower frequency range and the effects need to be investigated.

A fast prediction of the operating dispersion curve can be obtained by using the multi-mode coupling method, as shown in Fig. 2. The cut-off frequency of the $\mathrm{TE}_{21}$ mode is much smaller than the $\mathrm{TM}_{21}$ and $\mathrm{TE}_{22}$ modes, thus it will not contribute to the operating eigenwave in the frequency range of interest for the microwave compressor. The choice of the mean radius of the helically corrugated waveguide needs to balance the dispersion characteristic as well as the power capability. A smaller $R_{0}$ has a larger cut-off frequency gap between the $\mathrm{TM}_{21}, \mathrm{TE}_{31}$ and $\mathrm{TE}_{22}$ modes, thus it is possible to reduce the effect caused by the coupling between the $\mathrm{TE}_{31}$ and $\mathrm{TM}_{21}$ modes. However, a small radius can also reduce the power capability, which is opposite to the original aim. The axial period of the helical waveguide $d$ determines the intersection points of the coupled modes, and the corrugation depth $R_{1}$ greatly contributes to the coupling coefficients, a larger corrugation depth results in stronger coupling. An optimum set of parameters can be searched for simply by parameter sweeping, or from an optimization algorithm to find an optimum balance among the requirements, such as the power capability, the operating frequency range, and the dispersion characteristic of the operating eigenwave.

\section{Comparison Between the Calculation AND THE EXPERIMENT}

The five-fold helically corrugated waveguide was studied with dimensions of $R_{0}=32.84 \mathrm{~mm}, R_{1}=2.43 \mathrm{~mm}$, and $d=$ $33.26 \mathrm{~mm}$. The dispersion curve was also measured using a $65 \mathrm{GHz}$ vector network analyzer (VNA) (Anritsu 37397A). The experimental setup is shown in Fig. 4. From left to right, a rectangular to circular mode converter (1) in Fig. 4) was first 


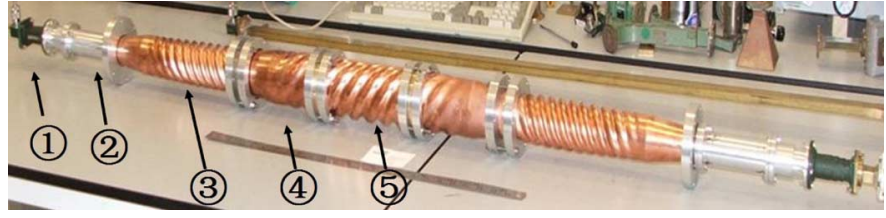

Fig. 4. Experimental setup for measuring the dispersion curve of the five-fold helical waveguide.

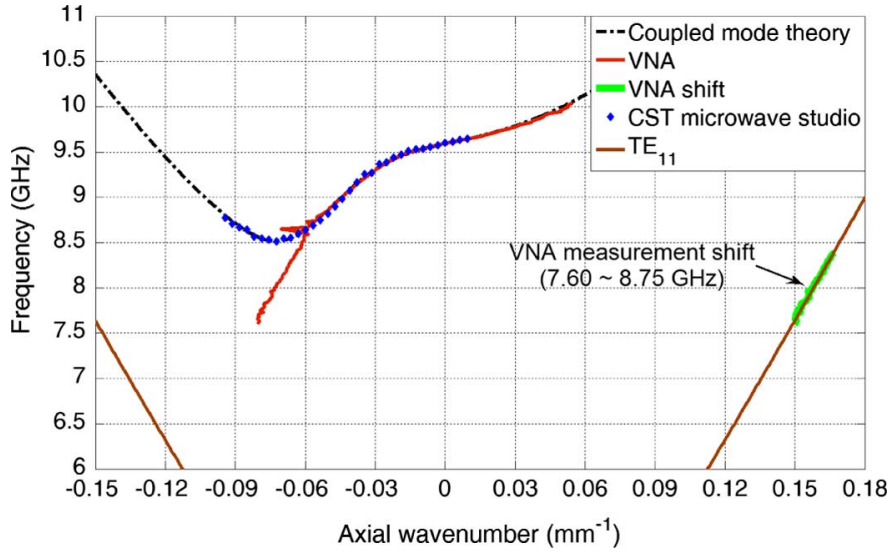

Fig. 5. Dispersion curve from coupled mode theory, CST Microwave Studio simulation, and the VNA measurement.

used to convert the fundamental $\mathrm{TE}_{10}$ mode in rectangular waveguide to a linearly polarized $\mathrm{TE}_{11}$ mode in the circular waveguide. This is followed by an elliptical polarizer (2) in Fig. 4) that converts the linearly polarized $\mathrm{TE}_{11}$ mode into a left hand rotating $\mathrm{TE}_{11}$ wave. A four-fold helical mode converter (3) in Fig. 4) was then used to convert the circularly polarized $\mathrm{TE}_{11}$ mode into a circularly polarized $\mathrm{TE}_{31}$ mode. The mode convertor was designed to have $>90 \%$ conversion efficiency in the frequency range of $8.75 \sim 10.0 \mathrm{GHz}$. Finally a five-fold helical taper (4) in Fig. 4) was used before introducing the $\mathrm{TE}_{31}$ mode into the five-fold helically corrugated waveguide (5) in Fig. 4) to make sure the $\mathrm{TE}_{31}$ mode smoothly converted to the operating eigenwave without reflection. On the other side of the helical waveguide, the same configuration was used.

Fig. 5 shows the dispersion curves calculated from the coupled mode theory, simulated from the eigensolver in CST Microwave Studio as well as measured by the VNA. From the coupled mode calculation, there are four eigenwaves resulting from the mode coupling between the $\mathrm{TE}_{31}$ and the $\mathrm{TE}_{21}$, the $\mathrm{TM}_{21}$, and the $\mathrm{TE}_{22}$ modes. Eigenwave 2 is the operating eigenwave for microwave compression. The dispersion curves from coupled mode theory, simulation using CST Microwave Studio and measurement using a VNA agree well with each other in the frequency range $8.75 \sim 10.0 \mathrm{GHz}$, as shown in Fig. 5 .

However the measured dispersion at the lower frequency band $(7.6 \sim 8.75 \mathrm{GHz})$ underwent a change of trend. This is due to the fact that the frequency is out of the operating band of the $\mathrm{TE}_{11}$-to- $\mathrm{TE}_{31}$ mode convertor. The dominant power in the helically corrugated waveguide is still in the $\mathrm{TE}_{11}$ mode in this lower frequency band. If the measured result in this frequency range is shifted by a factor of $2 \pi N / L$, where $N$ is an integer, and $L$ is the length of the helically corrugated waveguide in the measurement, it agrees well with the dispersion curve of the $\mathrm{TE}_{11}$ mode, as shown in Fig. 5. At a frequency of $8.6 \sim 8.8 \mathrm{GHz}$ a small content of the $\mathrm{TE}_{31}$ mode exists in the helical waveguide in conjunction with the $\mathrm{TE}_{11}$ mode, this causes the measured dispersion result in this frequency range to be spiky as it is the mixed phase information of the two modes.

\section{CONCLUSION}

In this paper, a detailed description of the multi-mode coupled wave theory for calculation of the dispersion characteristic of helically corrugated waveguide is given and it is applied to analyze a five-fold helical waveguide. This calculated result from the theory was found to be in good agreement with the CST Microwave Studio simulation result, as well as the VNA measurements.

\section{ACKNOWLEDGMENT}

The authors would like to thank V. L. Bratman, G. G. Denisov, S. V. Samsonov, and S.V. Mishakin for their helpful discussions.

\section{REFERENCES}

[1] A. S. Gilmour, Principles of Traveling Wave Tubes. Boston, MA: Artech House, 1994.

[2] , R. J. Barker, J. H. Booske, N. C. Luhmann, and S. N Gregory, Eds. , Modern Microwave and Millimeter-Wave Power Electronics. New York: IEEE, 2005.

[3] C. K. Chong, D. B. McDermott, M. M. Razegh, N. C. Luhmann, J. Pretterebner, D. Wagner, M. Thumm, M. Caplan, and B. Kulke, "Bragg reflectors," IEEE Trans. Plasma Sci., vol. 20, no. 3, pp. 393-402, Mar. 1992.

[4] N. Ginzburg, N. Peskov, A. Sergeev, A. D. R. Phelps, A. W. Cross, and I. V. Konoplev, "The use of a hybrid resonator consisting of one dimensional and two dimensional bragg reflectors for generation of spatially coherent radiation in a coaxial free-electron laser," Phys. Plasmas, vol. 9, no. 6, pp. 2798-2802, 2002.

[5] V. L. Bratman, A. W. Cross, G. G. Denisov, W. He, A. D. R. Phelps, K. Ronald, S. V. Samsonov, and C. G. W. A. R. Young, "High-gain wide-band gyrotron traveling wave amplifier with a helically corrugated waveguide," Phys. Rev. Lett., vol. 84, no. 12, pp. 2746-2749, 2000.

[6] A. W. Cross, W. He, A. D. R. Phelps, K. Ronald, C. G. Whyte, A. R. Young, C. W. Robertson, E. G. Rafferty, and J. Thomson, "Helically corrugated waveguide gyrotron traveling wave amplifier using a thermionic cathode electron gun," Appl. Phys. Lett., vol. 90, p. 253501, 2007.

[7] S. V. Samsonov, A. D. R. Phelps, V. L. Bratman, G. Burt, G. G. Denisov, A. W. Cross, K. Ronald, W. He, and H. Yin, "Compression of frequency-modulated pulses using helically corrugated waveguides and its potential for generating multigigawatt rf radiatione," Phys. Rev. Lett., vol. 92, p. 118301, 2004.

[8] G. Burt, S. V. Samsonov, A. D. R. Phelps, V. L. Bratman, K. Ronald, G. G. Denisov, W. He, A. R. Young, A. W. Cross, and I. V. Konoplev, "Microwave pulse compression using a helically corrugated waveguide," IEEE Trans. Plasma Sci., vol. 33, no. 2, pp. 661-667, Feb. 2005.

[9] W. He, A. W. Cross, A. D. R. Phelps, K. Ronald, C. G. Whyte, S. V. Samsonov, V. L. Bratman, and G. G. Denisov, "Theory and simulations of a gyrotron backward wave oscillator using a helical interaction waveguide," Appl. Phys. Lett., vol. 89, p. 091504, 2006.

[10] W. He, K. Ronald, A. R. Young, A. W. Cross, A. D. R. Phelps, C. G Whyte, E. G. Rafferty, J. Thomson, C. W. Robertson, D. C. Speirs, S. V. Samsonov, V. L. Bratman, and G. G. Denisov, "Gyro-BWO experiments using a helical interaction waveguide," IEEE Trans. Electron Devices, vol. 52, no. 5, pp. 839-844, May 2005. 
[11] G. G. Denisov and M. G. Reznikov, "Corrugated cylindrical resonators for short-wavelength relativistic microwave oscillators," Radiophys. Quantum Electron., vol. 25, no. 5, pp. 407-413, 1982.

[12] S. J. Cooke and G. G. Denisov, "Linear theory of a wide-band gyro-TWT amplifier using spiral waveguide," IEEE Trans. Plasma Sci., vol. 26, no. 3, pp. 519-530, Mar. 1998.

[13] G. G. Denisov, V. L. Bratman, A. D. R. Phelps, and S. V. Samsonov, "Gyro-TWT with a helical operating waveguide: New possibilities to enhance efficiency and frequency bandwidth," IEEE Trans. Plasma Sci., vol. 26, no. 3, pp. 508-518, Mar. 1998.

[14] G. Burt, S. V. Samsonov, K. Ronald, G. G. Denisov, A. R. Young, V. L. Bratman, A. D. R. Phelps, A. W. Cross, I. V. Konoplev, W. He, J. Thomson, and C. G. Whyte, "Dispersion of helically corrugated waveguides: Analytical, numerical, and experimental study," Phys. Rev. E, vol. 70, no. 4, p. 046402, 2004.

[15] L. Lewin, Theory of Waveguides: Techniques for the Solution of Waveguide Problems. London, U.K.: Newnes Butterworths, 1975.

[16] A. Nicolet and F. Zolla, "Finite element analysis of helicoidal waveguides," IET Sci., Meas., Technol., vol. 1, no. 1, pp. 67-70, 2007.

[17] J. L. Wilson, C. Wang, A. E. Fathy, and Y. W. Kang, "Analysis of rapidly twisted hollow waveguides," IEEE Trans. Plasma Sci., vol. 57, no. 1, pp. 130-139, Jan. 2009.

[18] B. Z. Katsenelenbaum, L. M. D. Ro, M. Pereyaslavets, M. S. Ayza, and M. K. A. Thumm, Theory of Non-Uniform Waveguides: The CrossSection Method. London, U.K.: The IET, 1999.

[19] M. McStravick, S. V. Samsonov, K. Ronald, S. V. Mishakin, W. He, G. G. Denisov, C. G. Whyte, V. L. Bratman, A. W. Cross, A. R. Young, P. MacInnes, C. W. Robertson, and A. D. R. Phelps, "Experimental results on microwave pulse compression using helically corrugated waveguide," J. Appl. Phys., vol. 108, no. 5, p. 054908, 2010.

[20] V. L. Bratman, G. G. Denisov, N. G. Kolganov, S. V. Mishakin, S. V. Samsonov, A. W. Cross, W. He, L. Zhang, M. McStravick, C. G. Whyte, A. R. Young, K. Ronald, C. W. Robertson, and A. D. R. Phelps, "Generation of $3 \mathrm{GW}$ microwave pulses in x-band from a combination of a relativistic backward-wave oscillator and a helical-waveguide compressor," Phys. Plasmas, vol. 17, p. 110703, 2010.

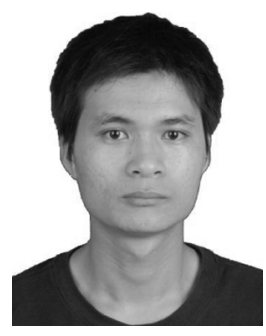

Liang Zhang received the B.Sc. degree in applied physics from the University of Science and Technology of China, Hefei, China, in 2004 and the M.Sc. degree in application of nuclear techniques from the China Academy of Engineering Physics, Chengdu, China, in 2007, respectively. He is currently pursuing the $\mathrm{Ph} . \mathrm{D}$. degree in physics from the Scottish Universities Physics Alliance, Department of Physics, University of Strathclyde, Glasgow, U.K.

His main research interests include pulse-power oscillators. technology, and Gyrotron-TWT/backward-wave

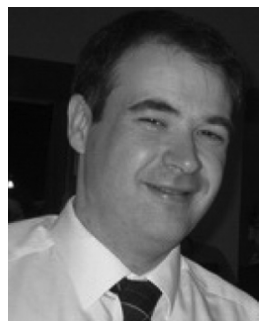

Kevin Ronald was born in Glasgow, U.K. He received the B.Sc. (with honors) and Ph.D. degrees in physics from the University of Strathclyde, Glasgow, U.K., in 1992 and 1997, respectively.

He is currently a Lecturer with the Scottish Universities Physics Alliance, Department of Physics, University of Strathclyde.

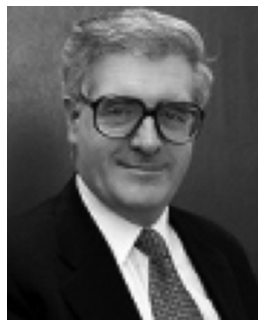

Alan D. R. Phelps was born in the U.K. in 1944. He received the B.A. degree (with honors) in physics and the M.A. degree from Cambridge University, Cambridge, U.K., in 1966 and 1970, respectively, and the D.Phil. degree for plasma research from Oxford University, Oxford, U.K., in 1970.

He has been with the University of Strathclyde, Glasgow, U.K., since 1978, where he initially founded a research group, became a Full Professor in 1993, and is currently with the Scottish Universities Physics Alliance, Department of Physics, where he was the Head of the department from 1998 to 2001. His research interests include high-power free-electron radiation sources and plasmas.

Dr. Phelps is a Fellow of the Institute of Physics and of the Royal Society of Edinburgh.

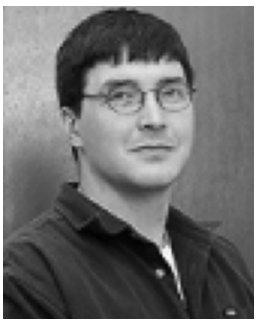

Colin G. Whyte was born in Selkirk, U.K., in 1969. He received the B.Sc. degree in physics from the University of Glasgow, Glasgow, U.K., in 1991 and the M.Sc. degree in laser physics and pulsed-power technology and the Ph.D. degree in physics from the University of St Andrews, St Andrews, U.K., in 1992 and 1996, respectively.

He has been with the University of Strathclyde, Glasgow, U.K., since 1996 in the ABP Group, Department of Physics, where he is also currently with SUPA. His research interests include the design and experimental investigation of high-power broadband microwave amplifiers and oscillators, including novel helical gyro-TWT devices and reverse-guide-field free-electron lasers. He also designs and builds high-voltage pulsed-power supplies.

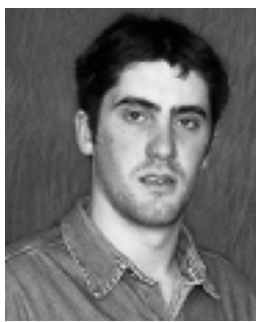

Craig W. Robertson was born in Ayrshire, U.K., in 1977. He received the B.Sc. (with honors) degree in physics and the Ph.D. degree from the University of Strathclyde, Glasgow, U.K., in 1999 and 2004, respectively.

He is currently with SUPA, Department of Physics, University of Strathclyde. His interests include lowtemperature plasma production and diagnostics, as well as high-power microwave sources.

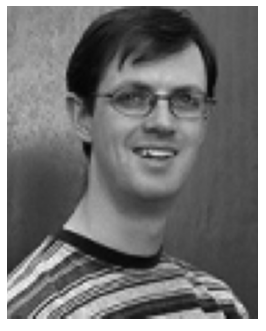

Alan R. Young received the B.Sc. degree in physics, the M.Sc. degree in information technologies systems, and the Ph.D. degree, with a dissertation on high-quality electron beams for CARMs, from the University of Strathclyde, Glasgow, U.K., in 1993, 1994, and 1998, respectively.

He has since continued to work as a Research Fellow with the Department of Physics, University of Strathclyde, on a variety of gyro-devices, including CARM and gyro-TWT, where he is also currently with SUPA.
Wenlong He received the B.Sc. degree in physics M.Sc. degree in accelerator physics from the China Academy of Engineering Physics, Chengdu, China, in 1988, and the Ph.D. degree in relativistic electron

He is currently a Senior Research Fellow the Scottish Universities Physics Alliance, Department of Physics, University of Strathclyde. His main research interests include relativistic electron beams, CARMs, FELs, Gyrotron-TWT/backward-wave oscillators, and other 


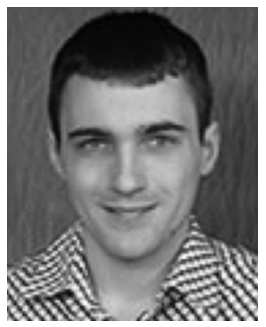

Craig R. Donaldson received the B.Sc. (with honors) degree in physics, the M.Sc. degree in high power RF and the Ph.D. degree in physics from the University of Strathclyde, Glasgow, U.K., in 2005, 2006, and 2009, respectively.

$\mathrm{He}$ is currently with SUPA, Department of Physics, University of Strathclyde. His main research interests include electron-beam generation, gyro-TWT/BWOs, and helically corrugated waveguides.

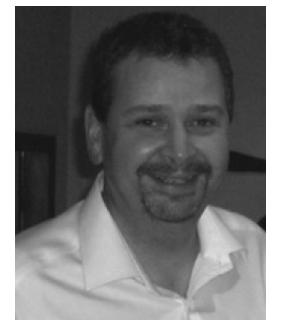

Adrian W. Cross received the B.Sc. degree (with honors) in physics and the Ph.D. degree from the University of Strathclyde, Glasgow, U.K., in 1989 and 1993, respectively.

He joined the Atoms, Beams, and Plasmas Group, University of Strathclyde, in 1993 initially as a Research Fellow and then as a Lecturer in 2000, Senior Lecturer in 2005, and is now a Reader with the Department of Physics. From 2002 to 2007, he was an Engineering and Physical Science (EPSRC) Advanced Fellow. He has been involved in various aspects of research on gyrotrons, cyclotron autoresonance masers, free-electron lasers, superradiant sources, gyrotron travelling wave amplifiers and plasma applications. More recently, he has primarily been concerned with research on microwave pulse compression, $\mathrm{THz}$ radiation sources and pseudospark physics. 